and possibly of certain higher invertebrates, in which fluid is filtered from blood vessels under pressure from the heart through colloid-retaining membranes (for example, tho glomerulus) into renal tubules.

Such an explanation is not applicable in the case of the Protozoa. During diastole, the contractile vacuole has no connexion with the exterior, and is completely surrounded by more or less fluid cytoplasm; this cytoplasm is in turn surrounded by the pellicle. Infiltration of flutid from the cytoplasm into the contractile vacuole cannot reduce the volume of substance enclosed within the pellicle; therefore tension on the pellicle, or in other words the hydrostatic pressure of the cytoplasm, cannot be the cause of infiltration.

This view is supported by my own observations. Sometimes when the marine ciliate Cothurnia is transferred to very dilute sea-water, it swells so violently that the pellicle bursts, and a blister is produced in which the cytoplasm is separated from the external medium only by a very thin protoplasmic membrane. It may be presumed that such a membrane could not withstand nearly so great a mechanical pressure as could the normal pellicle, and yet at first under these conditions the vacuole may increase in volume very rapidly; systole, however, is often ineomplete. Again, when a Cothurnia is transferred from a solution of lower to one of higher osmotic pressure, it shrinks and frequently the pellicle becomes wrinkled; and yet the contractile vacuole continues to function (although at a reduced rate).

The possibility of simple osmotic uptake of water into the contractile vacuole has already been discussed and discarded ${ }^{2}$. Both theoretical and practical considerations lead to the conclusion that during diastole water is forced into the contractile vacuole against the osmotic gradient by some unknown secretory process. No other hypothesis is consistent with the osmoregulatory theory of contractile vacuoles - a theory for which much evidence has already been presented ${ }^{2,3}$, and for which much additional support has now been obtained in experiments on freshwater Protozoa. It has been found that the contractile vacuoles of certain freshwater peritrich Ciliates are inhibited to a large extent by cyanide, and that this treatment is followed by a swelling of the body. The results so far obtained suggest that the contractile vacuole maintains a difference of osmotic pressure across the body surface approximately equivalent to a 0.05 molar solution of a non-electrolyte.

Further experiments are in progress.

Birkbeck Collegi',

J. A. KrTching.

London.

Dec. 13.

1 Picken, I. E. R., J. Exp. Biol., 13, 387 (1936),

${ }^{2}$ Kitching, J. A., J. Exp. Biol., 11, 364 (1934).

${ }^{3}$ Kitching, J. A., J. Exp. Biol., 13, 11 (1936).

\section{Inheritance of a Differential Growth-Ratio}

THE relation between the sizes of two growing organs of an animal or plant is given by the equation $y=t x^{k}$. where $y$ is the size of one organ at any given time, $x$ is the size of the other organ, $b$ is a constant, and $k$ is the differential growth-ratio. It can be readily shown that if $\alpha$ and $\beta$ are the relative growth-rates of the two organs, then $k=\alpha / \beta$. The application of this formula has so far been limited almost entirely to the study of animal growth, but we have recently employed it in the study of the growth of tomatoes. We have also obtained preliminary data on the mode of inheritance of the differential growth-ratio in the first filial generation.

Populations of a tall species of Lycopersicum (L. racemigerum), and a dwarf strain of the tomato (L. esculentum var. "Chinaman") were grown side by side with a population of first generation hybrids (L. rac. × "Chinaman") which exhibited marked heterosis. Dry weight samples were taken during the flowering period up to the twenty-third week after sowing. The differential growth-ratio between stem weight and leaf weight for each of the three strains was found to remain constant over the duration of the experiment, the following values being obtained : L. racemigerum $=1 \cdot 36$; "Chinaman" $=1.06 ; F_{1}$ hybrid $=1 \cdot 30 . k$ for the hybrid was not intermediate between the parental values but closely resembled that of the tall parent, so that it is inherited in the first generation in the manner of a simple dominant. Thus, in its mode of inheritance, the differential growth-ratio resembles the other physiological characteristics which have been investigated, namely, the efficiency index, assimilation rate and respiration rate.

Eric Ashby.

L. C. Luckwill.

Department of Botany,
University, Bristol.

\section{Permanganates and Plant Growth}

IN preliminary tests carried out during the last few years, it was observed that the application of potassium permanganate to various plants was followed by a marked growth response, and in this connexion a series of controlled pot experiments was carried out with the cactus Opuntia Leuchotricha in quartz sand.

Permanganates were much more effective than manganous salts, but both produced marked increases in growth. The uptake of minerals also depended largely on treatment. For example, a considerably greater absorption of manganese was obtained from manganous salt than from permanganates. It would appear, therefore, that the effect produced by permanganates is not wholly manurial.

Further work is in progress, and an account will be published shortly.

\section{E. Webster.}

IAN M. RoBERTSON.

Macaulay Institute for Soil Research, Craigiebuckler, Aberdeen.

Dec. 7.

\section{Stereochemistry of Bivalent Tin and Lead}

SEVERAL bivalent metals (nickel, palladium, platinum, copper and silver) are known to exhibit a planar distribution of valencies in their quadricovalent co-ordination compounds. On the other hand, it has been shown that, in certain derivatives of quadrivalent platinum and univalent copper and silver ${ }^{1}$, the four valencies of the metal atom have a tetrahedral arrangement, so that it appears highly 